\title{
Local stiffness and work function variations of hexagonal boron nitride on $\mathrm{Cu}(111)$
}

\author{
Abhishek Grewal $^{*}$, Yuqi Wang ${ }^{1,2}$, Matthias Münks ${ }^{1}$, Klaus Kern ${ }^{1,3}$ \\ and Markus Ternes ${ }^{*} 1,2,4$
}

\section{Letter}

\section{Address:}

${ }^{1}$ Max Planck Institut für Festkörperforschung, Heisenbergstrasse 1, D-70569 Stuttgart, Germany, ${ }^{2}$ Peter Grünberg Institute,

Forschungszentrum Jülich, D-52425 Jülich, Germany, ${ }^{3}$ Institut de Physique, École Polytechnique Fédérale de Lausanne, $\mathrm{CH}-1015$ Lausanne, Switzerland and ${ }^{4}$ II. Institute of Physics, RWTH Aachen University, D-52074 Aachen, Germany

Email:

Abhishek Grewal* - a.grewal@fkf.mpg.de;

Markus Ternes* - ternes@physik.rwth-aachen.de

* Corresponding author

Keywords:

decoupling layers; hexagonal boron nitride; local stiffness; Moiré superstructure; work function variation

\author{
Beilstein J. Nanotechnol. 2021, 12, 559-565. \\ https://doi.org/10.3762/bjnano.12.46 \\ Received: 30 March 2021 \\ Accepted: 31 May 2021 \\ Published: 17 June 2021 \\ Associate Editor: E. Meyer \\ (c) 2021 Grewal et al.; licensee Beilstein-Institut. \\ License and terms: see end of document.
}

\begin{abstract}
Combined scanning tunnelling and atomic force microscopy using a qPlus sensor enables the measurement of electronic and mechanic properties of two-dimensional materials at the nanoscale. In this work, we study hexagonal boron nitride ( $h$-BN), an atomically thin 2D layer, that is van der Waals-coupled to a $\mathrm{Cu}(111)$ surface. The system is of interest as a decoupling layer for functional 2D heterostructures due to the preservation of the $h$-BN bandgap and as a template for atomic and molecular adsorbates owing to its local electronic trapping potential due to the in-plane electric field. We obtain work function $(\Phi)$ variations on the $h$ - $\mathrm{BN} / \mathrm{Cu}(111)$ superstructure of the order of $100 \mathrm{meV}$ using two independent methods, namely the shift of field emission resonances and the contact potential difference measured by Kelvin probe force microscopy. Using 3D force profiles of the same area we determine the relative stiffness of the Moiré region allowing us to analyse both electronic and mechanical properties of the 2D layer simultaneously. We obtain a sheet stiffness of $9.4 \pm 0.9 \mathrm{~N} \cdot \mathrm{m}^{-1}$, which is one order of magnitude higher than the one obtained for $h$ - $\mathrm{BN} / \mathrm{Rh}(111)$. Using constant force maps we are able to derive height profiles of $h-\mathrm{BN} / \mathrm{Cu}(111)$ showing that the system has a corrugation of $0.6 \pm 0.2 \AA$, which helps to demystify the discussion around the flatness of the $h$ - $\mathrm{BN} / \mathrm{Cu}(111)$ substrate.
\end{abstract}

\section{Introduction}

Two-dimensional hexagonal boron nitride $(h-\mathrm{BN})$ is among the list of materials that garnered tremendous interest following the exfoliation of mono- and few-layer thick graphene films [1,2].
Unique properties, such as high thermal stability and conductivity, immense intra-sheet stiffness, and excellent dielectric properties, make $h$-BN interesting for technological applica- 
tions. For example, thin films of $h$-BN have been used as a passivating layer for graphene and $\mathrm{MoS}_{2}$-based electronics utilising the small lattice mismatch, the large optical phonon modes, and particularly the large bandgap [3-10]. Furthermore, when grown on metal substrates $h$-BN can be used as a nanoscale template for atoms, molecules, and nanostructures with well-controlled adsorption and electronic properties [11-18]. In such systems, $h$-BN shows a rich structural and electronic morphology, which depends on the lattice mismatch and the interaction strength with the substrate: Large and flat lattice-matched terraces for $h$-BN/Ni(111) [19,20], strain-induced highly corrugated layers for $h-\mathrm{BN} / \mathrm{Rh}(111)$ [21-23], and template layers for molecules with strong local variations of the work function for $h$-BN/Ir(111) [24] are representative of such morphological diversity.

We use low-temperature combined scanning tunnelling (STM) and non-contact atomic force microscopy (nc-AFM) to study $h$-BN on $\mathrm{Cu}(111)$. This template has interesting properties because the dielectric layer is only very weakly bound to the metal and shows an electronically induced Moiré superstructure $[25,26]$. First STM studies on this system pointed to only a small geometrical corrugation [27]. Further experimental investigations, using both local probes and averaging techniques, revealed more details of the mechanical and electronic properties of the system, but also inconsistent results about the structural corrugation [26,28-30]. For example, Brülke et al. used high-resolution low-energy electron diffraction and normal incidence X-ray standing wave techniques to detect the large separation of $3.24 \AA$ between the $h$-BN sheet and the topmost $\mathrm{Cu}(111)$ layer [29]. They found almost no height difference between $\mathrm{B}$ and $\mathrm{N}$ atoms and excluded significant buckling perpendicular to the surface. Interestingly, this is in contrast to measurements by Schwarz et al. who used a more local analysis of the corrugation by exploiting nc-AFM concluding an absolute height difference of 0.3-0.7 ̊ between "rim" and "valley" sites of the spatially corrugated monolayer [26]. Recently, however, Zhang et al. used STM in combination with DFT simulations to study the variation of the local work function and bandgap within the Moire superlattice and found that the variation depends on the angle of the Moire with respect to the substrate lattice, but inferred only marginal structure modulation [30].

To shed more light on this controversy we use an alternative method to verify the mechanical properties of the monolayer by measuring the stiffness of the $h$-BN layer at different locations of the superstructure and comparing these results with concomitantly recorded local work function variations. We determine the stiffness of the system by mapping and comparing the shortrange interaction forces between the monolayer and the probing metallic tip [31]. This technique enables us to detect the sheet stiffness with unprecedented spatial resolution [23]. On $h$-BN/Rh(111), a different system than the one studied in this work, the extremely low stiffness of only approx. $1 \mathrm{~N} \cdot \mathrm{m}^{-1}$ at the weakly bound rim areas confirmed the buckling of the monolayer into the third dimension to relieve the strain induced by the significant lattice mismatch of this strongly corrugated van der Waals layer [23].

\section{Results and Discussion STM/AFM on $h-\mathrm{BN} / \mathrm{Cu}(111)$}

As illustrated in Figure 1a, we employ nc-AFM to probe the electronic and topographic structure of a monolayer of $h$-BN on a $\mathrm{Cu}(111)$ surface. Figure $1 \mathrm{~b}$ shows a typical large-scale constant-current STM scan of this structure. We observe the monolayer growing over step edges of the underlying $\mathrm{Cu}(111)$ substrate. Weak interlayer interaction allows the van der Waals layer to have varying relative rotational orientations, $\theta=0-4^{\circ}$, on the substrate corresponding to a Moiré pattern wavelength of $\lambda=3-14 \mathrm{~nm}$. Furthermore, we observe an upward shift of the surface state onset of $\mathrm{Cu}(111)$ from approx. $-455 \mathrm{meV}$ on the bare substrate to approx. $-320 \mathrm{meV}$ on $h-\mathrm{BN} / \mathrm{Cu}(111)$ (Figure 1c) [32]. We found this shift to vary only marginally (approx. $\pm 10 \mathrm{meV}$ ) with the Moiré periodicity or between rim and valley sites $[33,34]$.
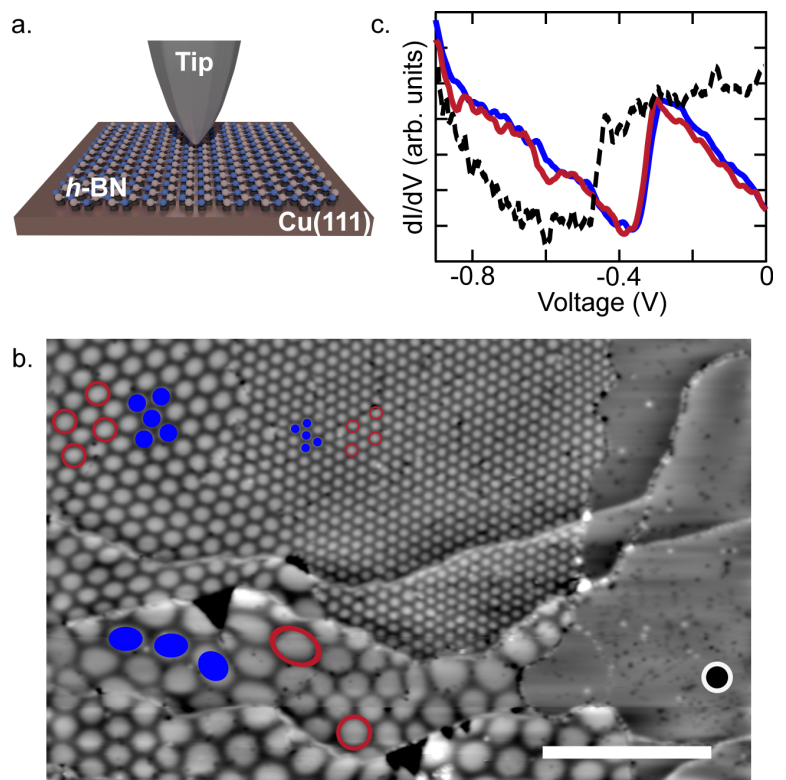

Figure 1: (a.) Scheme of the experiment. (b.) Large-scale $\left(200 \times 125 \mathrm{~nm}^{2}\right)$ constant-current $(I=20 \mathrm{pA}, V=3.7 \mathrm{~V})$ STM topography of $h-\mathrm{BN} / \mathrm{Cu}(111)$ and the bare $\mathrm{Cu}(111)$ surface. Blue circles and red rings mark exemplary valley and rim areas, respectively.

(c.) Differential conductance $\mathrm{d} / / \mathrm{d} V$ spectra taken at rim (red line) and valley (blue line) sites and at the bare $\mathrm{Cu}(111)$ substrate (dashed black line). 
$h$ - $\mathrm{BN} / \mathrm{Cu}(111)$ is known to have an indirect bandgap of $6.1 \mathrm{eV}$ [35], which can be modulated by the Moiré pattern [30]. We analyse the substrate using STM topography, $\mathrm{dI} / \mathrm{d} V$, and frequency shift, $\Delta f$, AFM maps under low (in-gap) and high (conduction band onset) bias conditions (see Figure 2). Due to $h$-BN being insulating, no spectroscopic contribution is expected at low bias voltages making it transparent to STM, as seen in Figure 2b,d. At this bias, only Friedel oscillations due to the scattering of the $\mathrm{Cu}(111)$ surface-state electrons on defects and adsorbates are observed. Contrarily, as Figure 2a reveals, at higher bias, the STM topography corresponds to the modulation of the $h-\mathrm{BN} / \mathrm{Cu}(111)$ interface state as we will show below.
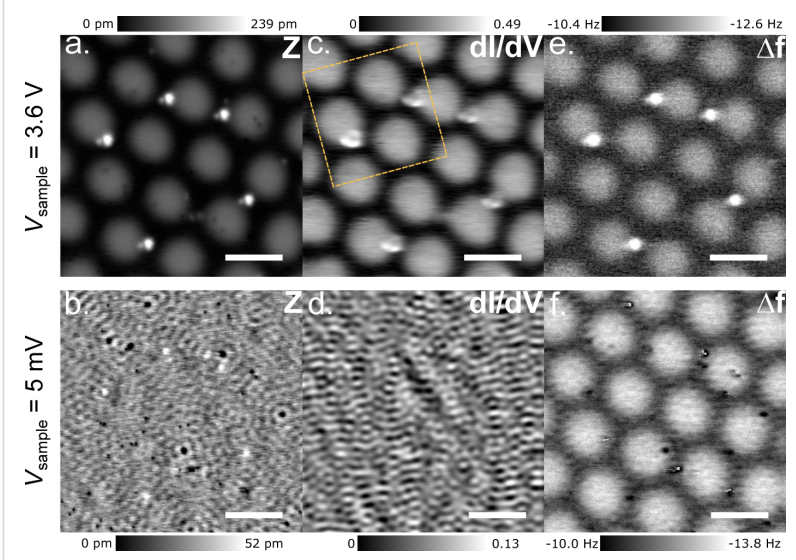

Figure 2: STM/AFM characterisation of a $h-\mathrm{BN} / \mathrm{Cu}(111)$ Moiré superstructure. (a., b.) Constant-current topography at $I=500 \mathrm{pA}$ and $V=3.6 \mathrm{~V}$ (top) or $V=5 \mathrm{mV}$ (bottom). (c., d.) Simultaneously measured differential conductance $(\mathrm{d} / / \mathrm{d} V)$ maps $\left(V_{\bmod }=10 \mathrm{mV}\right.$ (top) and $V_{\text {mod }}=1 \mathrm{mV}$ (bottom). (e., f.) Frequency shift $(\Delta f)$ maps

$\left(A_{\text {osc }}=50 \mathrm{pm}\right)$. The dashed yellow box marks the area used for the $\Phi$ maps in Figure 3 (see below). Scale bar: $10 \mathrm{~nm}$.

Despite the large change in electronic density of states and, thus, tip height between the data obtained at the two different sample biases, we observe a one-to-one correspondence between the simultaneously recorded $\Delta f$ images and the STM topography. Also, the $\Delta f$ variation between rim and valley areas in both images changes only marginally. The additionally imaged adsorbates (dots or ring-like features) allow, thereby, the precise alignment between the subsequently acquired data sets.

\section{Work function variation}

While the work function is generally discussed in the framework of a macroscopic quantity [36], we will use the notation, valid also on the nanoscale, that $\Phi$ is the local surface potential measured from the Fermi level, $E_{\mathrm{F}}$ [37]. For a nanoscale patterned surface, such as $h-\mathrm{BN} / \mathrm{Cu}(111), \Phi$ fluctuations can originate from a locally varying charge transfer between the substrate and the dielectric layer [38-40].
In our studied substrate, it is the lattice mismatch between $h$-BN and the $\mathrm{Cu}(111)$ substrate that leads to a varying atomic registry and subsequently induces a lateral modulation of the charge transfer [41]. Additionally, this leads to in-plane electric fields, which have been shown to trap atoms, molecules, and nanoclusters $[11,13,42]$.

To map the local $\Phi$ fluctuations and to correlate them with the structural properties of the surface, we use two complementary methods: The first method is based on a shift of the field emission resonance (FER) induced by $\Phi$ variations. The effective potential well of depth $\Phi$ at the surface of a metal can accommodate a series of Rydberg states, extending a few angstroms into the vacuum above the metal surface [43]. These image potential states (IPSs), states arising from the long-range image potential experienced by an electron in front of a metal surface, are delocalised in the surface plane and contain the full band of the $2 \mathrm{D}$ electron gas. However, the electric field exerted by the proximity of the probing tip distorts the energy spacing of the IPSs. These distorted IPSs are referred as FER, which are revealed in $\mathrm{dI} / \mathrm{d} V$ measurements as strong peaks at positive bias. [43]. Figure 3a shows such spectra in which we observe a series of peaks whose energies are strongly influenced by the measurement position. The non-trivial double peak structure at $3.5-4.5 \mathrm{~V}$ is due to varying contributions from the two interfaces of the dielectric layer. We therefore evaluate the unambiguous shift of the second peak at around 5.6-6.0 V as a measure for the local $\Phi$ variation.

Our nc-AFM allows us to employ with Kelvin probe force microscopy (KPFM) a second, independent method to detect the variation in $\Phi$. For this we record the frequency shift, $\Delta f$, of the resonance frequency of the cantilever oscillating perpendicular to the surface as a function of the bias voltage (see Figure $3 b$ ). At the extrema of the parabolic $\Delta f$ curves, the electrostatic force is minimised by the applied voltage, which compensates the contact potential difference between $\Phi$ of the tip and $\Phi$ of the sample [44].

Using the shift of the FER we find an average variation between valley and rim regions of $\Delta \Phi=148 \pm 17 \mathrm{meV}$, which agrees well with previous observations [27,45]. Interestingly, however, we find a significantly smaller average difference between valley and rim regions of only $\Delta \Phi=86 \pm 16 \mathrm{meV}$ when analysing the contact potential difference (CPD) data. This hints toward a lower lateral resolution of the KPFM measurement compared to the FER map. The $\Delta f$ signal in KPFM originates from the relatively long-ranged electrostatic interaction, which is therefore a weighted average over the relevant size of the tip (radii of 5-10 nm [46]). It is of the same order as the size of the rim and valley regions and, as a result, leads to an underestima- 
a.
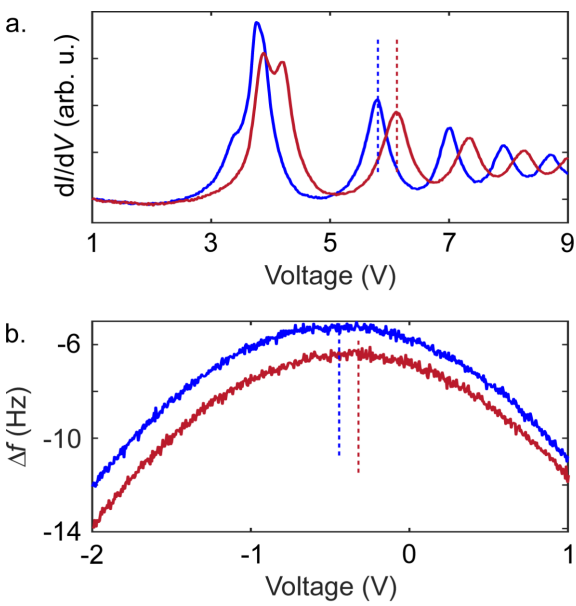

c.

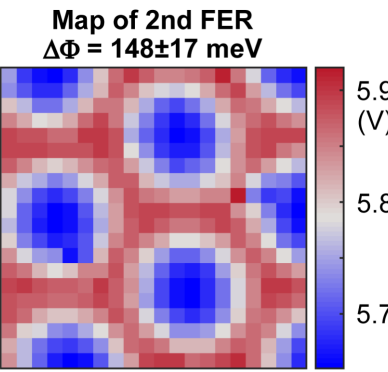

d.

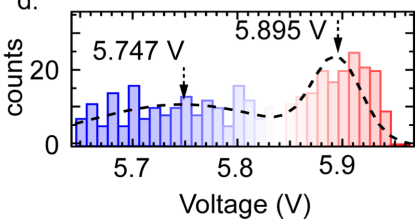

V)

5.8 e. Map of CPD using KPFM $\Delta \Phi=86 \pm 16 \mathrm{meV}$

$-0.64$

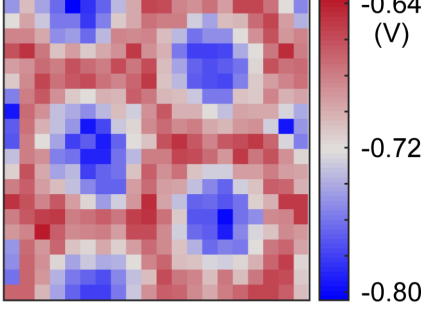

f.

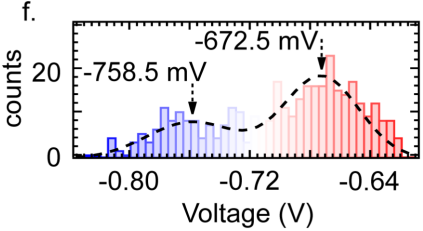

Figure 3: Work function variation between rim (red) and valley (blue) areas measured using (a.) d $/ \mathrm{d} V$ at constant current $(I=100 \mathrm{pA}$ ) and (b.) KPFM at constant height (stabilised in the valley at $I=100 \mathrm{pA}, V=10 \mathrm{mV}, A_{\mathrm{osc}}=50 \mathrm{pm}$ ), respectively. The dotted vertical lines mark exemplary FER and $\mathrm{CPD}$ values used for the spatially resolved plots shown in (c.) and (e.). The maps are recorded at the yellow box indicated in Figure $2 \mathrm{c}$ on a $20 \times 20$ grid over $20 \times 20 \mathrm{~nm}^{2}$. They display the position of the maximum of the second peak in the FER (c.) and the maximum of the KPFM parabola (e.), respectively. (d., f.) Histograms and fits for rim and valley where arrows mark the centre positions of the Gaussians used for the determination of the distribution centre.

tion of $\Delta \Phi$. Nevertheless, both measurement techniques agree well in their qualitative results as it is evident from the $\Delta \Phi$ maps (see Figure 3c,e).

\section{Stiffness}

Probing the force perpendicular to the substrate, $F_{\perp}$, at varying tip-sample separations $z$, the effective stiffness of a nanostructure can be evaluated by comparing the $F_{\perp}(z)$ behaviour at different areas of the Moiré superstructure [23]. Additionally, such a set of data enables us to obtain maps of constant tip-sample interaction forces that allow for the quantification of the corrugation of the Moiré superstructure.

To obtain such data we map the $\Delta f$ signal at constant oscillation amplitude for an $8 \times 8 \mathrm{~nm}^{2}$ area at 28 relative tip-surface distances. From the set point, $I=100 \mathrm{pA}, V=$ $10 \mathrm{mV}$, we approach the tip by $250 \mathrm{pm}$ (defined as $z=0 \mathrm{pm}$ ), set $V=0 \mathrm{mV}$, and record $\Delta f$ maps as the tip is retracted from $z=$ 0 to $270 \mathrm{pm}$, with a $10 \mathrm{pm}$ spacing (grey shaded area in Figure $4 b$ ).
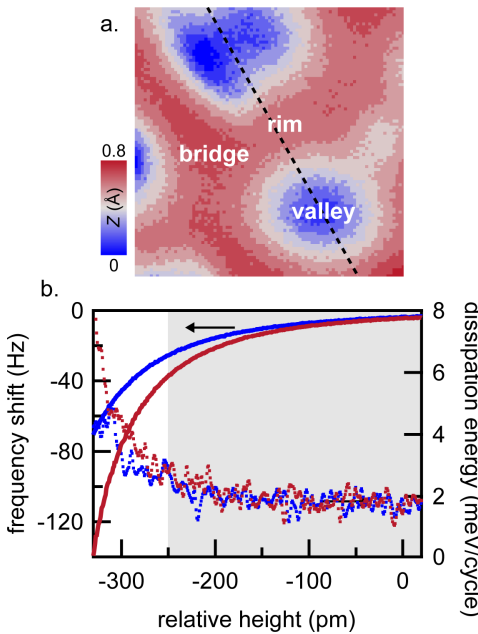

c.
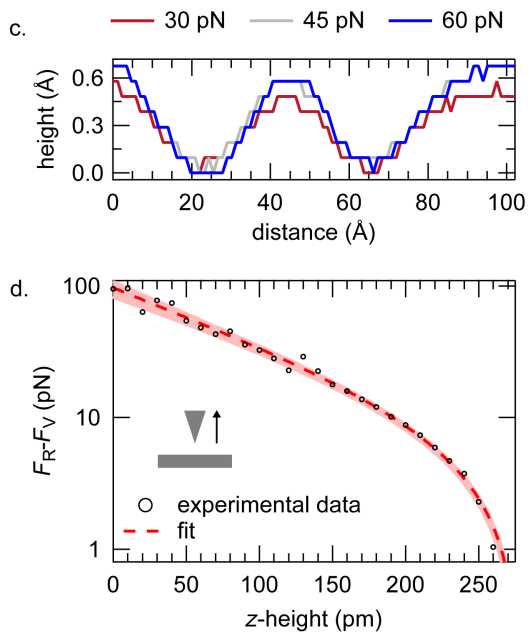
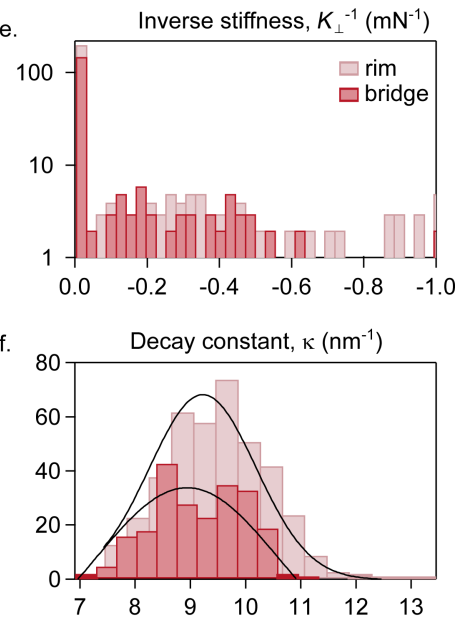

Figure 4: Local stiffness of $h$-BN/Cu(111). (a.) Topography of an $8 \times 8 \mathrm{~nm}^{2} h-\mathrm{BN} / \mathrm{Cu}(111)$ area corresponding to a constant force $F_{\perp}=30 \mathrm{pN}$. (b.) Point $\Delta f$ and excitation energy vs relative $z$-height curve obtained at rim (red) and valley (blue) positions. The grey shaded area marks the $z$-range used for the $\Delta f$ maps. (c.) Line profiles taken from constant-vertical-force maps along the black dashed line in (a.), at $F_{\perp}=30 \mathrm{pN}$ (red), $45 \mathrm{pN}$ (grey), and $60 \mathrm{pN}$ (blue), respectively. (d.) Average attractive short-range force obtained for the rim and the bridge region after subtracting the contribution from valley area (experimental data) and fit. The red area marks the $90 \%$ confidence range. (e., f.) Histograms of inverse stiffness $\left(K_{\perp}^{-1}\right)$ and decay constant ( $\mathrm{k}$ ) of the rim (pink) and bridge sites (red), respectively. 
Using the matrix inversion method [47], we convert the 3D stack of $\Delta f$ data into the out-of-surface force component $F_{\perp}$. The now obtained 3D force stack enables us to evaluate the interaction between the tip and the monolayer substrate without being strongly influenced by the electronic corrugation as in STM-only measurements. By taking a 2D cut at constant force through the 3D stack, we obtain a topography at a constant tip-substrate interaction force, which allows us to visualise the corrugation between rim and valley areas (see Figure 4a). Figure $4 \mathrm{c}$ shows different line profiles corresponding to constant force values of $F_{\perp}=30,45$, and $60 \mathrm{pN}$. These line profiles reveal an average corrugation of $0.6 \pm 0.2 \AA$, which agrees well with the corrugation of $0.3-0.7 \AA$ obtained by Schwarz and co-workers [26]. In these line profiles we obtain a minimal corrugation increase at increased constant force values, which hints to some mechanical relaxations of the rim areas under the influence of the force exerted by the tip.

To analyse this effect we separate the short-range forces, which act between the tip apex and the sample and which vary over the corrugation of the monolayer, from electrostatic and van der Waals long-range forces by subtracting the average total force $F_{\mathrm{V}}$ measured in the valley areas (blue regions in Figure $4 \mathrm{a}$ ) from the total forces $F_{\mathrm{R}}$ acting at rim and bridge sites of the superstructure (red regions in Figure 4a), assuming infinite stiffness for valley areas. An example of $\Delta f$-vs-z point spectra obtained at valley and rim regions is shown in Figure $4 \mathrm{~b}$. The grey shaded area, showing little change in dissipation, is used for the $z$-range for which $\Delta f$ maps are acquired. The resulting difference $F_{\mathrm{D}}=F_{\mathrm{R}}-F_{\mathrm{V}}$ is the additional short-range force, which solely influences rim and bridge areas and which might locally lift the $h$-BN layer leading to an increase of corrugation. Figure $4 \mathrm{~d}$ shows $F_{\mathrm{D}}(z)$ averaged over rim and bridge sites, which decays with $z$ mainly exponentially as expected from an interatomic short-range force when neglecting Pauli repulsion $[23,48]$. The over-exponential decay at $z>200 \mathrm{pm}$ is caused by a small offset of $F_{0} \approx 1 \mathrm{pN}$ due to the finite set of $\Delta f$ data, which results in $F_{\mathrm{R}} \equiv F_{\mathrm{V}}$ at the last measurement height $(z=270 \mathrm{pm}$, see Experimental section). A very soft $h$-BN layer would show an additional over-exponential increase at small $z$ due to the lifting of the sheet by $\Delta z=F_{\mathrm{D}}(z) K_{\perp}^{-1}$, where $K_{\perp}$ is the local vertical stiffness [23]. Assuming an exponential decay of the intrinsic short-range force between tip an substrate and compensating for any lifting, we get for the local vertical force, $F_{\mathrm{D}}$, as a function of relative height:

$$
F_{\mathrm{D}}(z)=\left(\kappa / K_{\perp}\right) \times W_{0}\left[F_{0} \kappa / K_{\perp} \exp (-\kappa z)\right]+F_{0},
$$

where $W_{0}$ is the real-valued branch of the Lambert $W$ function and $\kappa$ is the decay constant [23]. As shown in Figure 4d, we obtain a good agreement between our data and the model. The best fit yields an average local vertical stiffness of $K_{\perp}=9.4 \pm 0.9 \mathrm{~N} \cdot \mathrm{m}^{-1}$ (Figure $4 \mathrm{~d}$ ). This demonstrates the high stiffness (negligible softness) of the $h$-BN monolayer on $\mathrm{Cu}(111)$, which is one order of magnitude higher than that found on $\mathrm{Rh}$ (111) [23]. The statistical evaluation of the spatial variation of $K_{\perp}$ is shown in Figure 4e. The dramatic peak at small inverse stiffness in both rim and bridge areas means an almost perfect exponential behaviour of the short-range force and that $h-\mathrm{BN} / \mathrm{Cu}(111)$ undergoes no significant deformation. Also, the histogram of the decay constant $\kappa$ in Figure $4 \mathrm{f}$ reveals only negligible differences between $\operatorname{rim}\left(\kappa=9.2 \pm 1.3 \mathrm{~nm}^{-1}\right)$ and bridge areas $\left(\kappa=8.9 \pm 1.4 \mathrm{~nm}^{-1}\right)$ indicating almost no difference in the mechanical properties between different areas of the Moiré superstructure. Additionally, the cluster of $K_{\perp}^{-1}$ values close to $0 \mathrm{~m} \cdot \mathrm{N}^{-1}$ suggests that the short range forces between rim and bridge sites are almost identical.

\section{Conclusion}

We report the electronic and mechanical characterisation of $h$-BN/Cu(111) using an STM/AFM. Our STM studies corroborate that the $h$-BN monolayer is only weakly coupled to the $\mathrm{Cu}(111)$ surface as is evidenced by the large angular range of Moiré superstructures observed, which in turn leads to work function patterning. Using FER and KPFM maps we report a work function variation of $148 \pm 17$ and $86 \pm 16 \mathrm{meV}$, respectively, which agrees well with the previous experimental and theoretical studies $[27,45]$.

3D force maps, obtained via constant-height $\Delta f$ imaging, allow us to test the mechanical stability of the monolayer substrate in the short-range force regime. Using the AFM tip as a nanoindenter we probe its effect on the $h-\mathrm{BN} / \mathrm{Cu}(111)$ system. We obtain a sheet stiffness of $K_{\perp}=9.4 \pm 0.9 \mathrm{~N} \cdot \mathrm{m}^{-1}$, which is one order of magnitude larger than that obtained on $h-\mathrm{BN} / \mathrm{Rh}(111)$, indicating substantial mechanical stability. The small lattice mismatch between $h$-BN and $\mathrm{Cu}(111)$, compared to $h$-BN and $\mathrm{Rh}(111)$, results in lower strain and no buckling of the substrate and, thus, to high stiffness. Furthermore, our results corroborate that $h-\mathrm{BN} / \mathrm{Cu}(111)$ has a small corrugation of $0.6 \pm 0.2 \AA$ but is mechanically stiff making it an appealing platform for studying intrinsic electronic and mechanical properties of nanostructures.

\section{Experimental}

We employ a custom-built ultrahigh-vacuum (below $10^{-10} \mathrm{mbar}$ ) low-temperature $(T=1.4 \mathrm{~K}) \mathrm{nc}-\mathrm{AFM}$ operated in frequency-modulated mode. A stiff qPlus cantilever design [49] $\left(k_{0}=1800 \mathrm{~N} \cdot \mathrm{m}^{-1}, f_{0}=29077 \mathrm{~Hz}, Q=60000\right)$ at an oscillation amplitude $A_{\text {osc }}=50 \mathrm{pm}$ enables the nc-AFM functionality. We calibrated the amplitude prior to the measurement atop the bare 
$\mathrm{Cu}(111)$ substrate [50]. The bias voltage $V$ is applied to the substrate and the tunnelling current $I$ is measured at the virtually grounded tip. The STM/AFM images were processed with the Gwyddion software [51].

FER and KPFM measurements: FER measurements are taken by modulating $V\left(f_{\mathrm{m}}=607 \mathrm{~Hz}, V_{\mathrm{m}}=10 \mathrm{mV}\right.$ peak-to-peak $)$ and detecting the $\mathrm{d} I / \mathrm{d} V$ signal with the lock-in technique while the tip height is adjusted so that the current $I$ remains constant (constant-current mode) during the bias sweep. For KPFM measurements we stabilise the tip height at $I=100 \mathrm{pA}$ and $V=10 \mathrm{mV}$. We then record the frequency shift $\Delta f$ with respect to $f_{0}$ while $V$ is swept at constant tip height.

Vertical stiffness: The $3 \mathrm{D} \Delta f$ data $\left(8 \times 8 \times 0.27 \mathrm{~nm}^{3}\right)$, evaluated in this work, are obtained by taking $282 \mathrm{D}$ maps at successively increased tip-sample separation $(\Delta z=10 \mathrm{pm})$ starting from a tip height stabilised at $I=100 \mathrm{pA}, V=10 \mathrm{mV}$ in the valley area and approaching the tip by $-250 \mathrm{pm}$ at $V=0 \mathrm{mV}$. We define this $z$-height as $z=0$. We use the known exponential dependence of the average current on the tip retraction to compensate for any z-drift over the approx. $23 \mathrm{~h}$ of data acquisition.

Sample preparation: $\mathrm{A} \mathrm{Cu}(111)$ single crystal (MaTeck $\mathrm{GmbH}$ ) is cleaned via repeated cycles of Ar-ion sputtering at room temperature followed by annealing to $1020 \mathrm{~K}$ in an ultrahigh-vacuum preparation chamber. A partial layer of $h$-BN is grown by chemical vapour deposition by heating the $\mathrm{Cu}(111)$ sample to $980 \mathrm{~K}$ and exposing it to $25 \mathrm{~L}$ of borazine $\left(\mathrm{HBNH}_{3}\right)$ gas (Katchem spol s.r.o.). $h$-BN grows in a self-terminating growth process [19]. It is then transferred in situ to the nc-AFM for characterisation.

\section{Acknowledgements}

We would like to thank Prof. Dr. Willi Auwärter for stimulating discussions.

\section{Funding}

A. G., M. M., and M. T. acknowledge support from the Collaborative Research Center (SFB) 767 of the German Research Foundation (DFG) for financial support. M. T. acknowledges support by the Heisenberg Program (Grant No. TE 833/2-1) and the Priority Programme (SPP) 2244 of the DFG and Y. W. would like to thank support from Alexander von Humboldt Foundation through a Humboldt Research Fellowship for Postdoctoral Researchers.

\section{ORCID ${ }^{\circledR}$ iDs}

Abhishek Grewal - https://orcid.org/0000-0002-4566-8499

Markus Ternes - https://orcid.org/0000-0001-6800-4129

\section{Preprint}

A non-peer-reviewed version of this article has been previously published as a preprint: https://doi.org/10.3762/bxiv.2021.30.v1

\section{References}

1. Novoselov, K. S.; Geim, A. K.; Morozov, S. V.; Jiang, D.; Zhang, Y.; Dubonos, S. V.; Grigorieva, I. V.; Firsov, A. A. Science 2004, 306, 666-669. doi:10.1126/science.1102896

2. Novoselov, K. S.; Mishchenko, A.; Carvalho, A.; Castro Neto, A. H. Science 2016, 353, aac9439. doi:10.1126/science.aac9439

3. Watanabe, K.; Taniguchi, T.; Kanda, H. Nat. Mater. 2004, 3, 404-409. doi:10.1038/nmat1134

4. Dean, C. R.; Young, A. F.; Meric, I.; Lee, C.; Wang, L.; Sorgenfrei, S.; Watanabe, K.; Taniguchi, T.; Kim, P.; Shepard, K. L.; Hone, J. Nat. Nanotechnol. 2010, 5, 722-726. doi:10.1038/nnano.2010.172

5. Xue, J.; Sanchez-Yamagishi, J.; Bulmash, D.; Jacquod, P.; Deshpande, A.; Watanabe, K.; Taniguchi, T.; Jarillo-Herrero, P.; LeRoy, B. J. Nat. Mater. 2011, 10, 282-285. doi:10.1038/nmat2968

6. Britnell, L.; Gorbachev, R. V.; Jalil, R.; Belle, B. D.; Schedin, F.; Mishchenko, A.; Georgiou, T.; Katsnelson, M. I.; Eaves, L.; Morozov, S. V.; Peres, N. M. R.; Leist, J.; Geim, A. K.; Novoselov, K. S.; Ponomarenko, L. A. Science 2012, 335, 947-950. doi:10.1126/science.1218461

7. Pakdel, A.; Bando, Y.; Golberg, D. Chem. Soc. Rev. 2014, 43 , 934-959. doi:10.1039/c3cs60260e

8. Li, Q.; Zou, X.; Liu, M.; Sun, J.; Gao, Y.; Qi, Y.; Zhou, X.; Yakobson, B. I.; Zhang, Y.; Liu, Z. Nano Lett. 2015, 15, 5804-5810. doi:10.1021/acs.nanolett.5b01852

9. Cui, X.; Lee, G.-H.; Kim, Y. D.; Arefe, G.; Huang, P. Y.; Lee, C.-H.; Chenet, D. A.; Zhang, X.; Wang, L.; Ye, F.; Pizzocchero, F.; Jessen, B. S.; Watanabe, K.; Taniguchi, T.; Muller, D. A.; Low, T.; Kim, P.; Hone, J. Nat. Nanotechnol. 2015, 10, 534-540. doi:10.1038/nnano.2015.70

10. Mendelson, N.; Xu, Z.-Q.; Tran, T. T.; Kianinia, M.; Scott, J.; Bradac, C.; Aharonovich, I.; Toth, M. ACS Nano 2019, 13, 3132-3140. doi:10.1021/acsnano.8b08511

11. Bose, S.; García-García, A. M.; Ugeda, M. M.; Urbina, J. D.; Michaelis, C. H.; Brihuega, I.; Kern, K. Nat. Mater. 2010, 9, 550-554. doi:10.1038/nmat2768

12. Kahle, S.; Deng, Z.; Malinowski, N.; Tonnoir, C.; Forment-Aliaga, A.; Thontasen, N.; Rinke, G.; Le, D.; Turkowski, V.; Rahman, T. S.; Rauschenbach, S.; Ternes, M.; Kern, K. Nano Lett. 2012, 12, 518-521. doi:10.1021/nl204141z

13. Joshi, S.; Bischoff, F.; Koitz, R.; Ecija, D.; Seufert, K.; Seitsonen, A. P.; Hutter, J.; Diller, K.; Urgel, J. I.; Sachdev, H.; Barth, J. V.; Auwärter, W. ACS Nano 2014, 8, 430-442. doi:10.1021/nn406024m

14. Jacobson, P.; Herden, T.; Muenks, M.; Laskin, G.; Brovko, O.; Stepanyuk, V.; Ternes, M.; Kern, K. Nat. Commun. 2015, 6, 8536. doi:10.1038/ncomms9536

15. Muenks, M.; Jacobson, P.; Ternes, M.; Kern, K. Nat. Commun. 2017, 8, 14119. doi:10.1038/ncomms 14119

16. Will, M.; Atodiresei, N.; Caciuc, V.; Valerius, P.; Herbig, C.; Michely, T. ACS Nano 2018, 12, 6871-6880. doi:10.1021/acsnano.8b02127 
17. Mehler, A.; Néel, N.; Kröger, J. J. Phys. Chem. Lett. 2020, 11, 5204-5211. doi:10.1021/acs.jpclett.0c01320

18. Riss, A.; Richter, M.; Paz, A. P.; Wang, X.-Y.; Raju, R.; He, Y.; Ducke, J.; Corral, E.; Wuttke, M.; Seufert, K.; Garnica, M.; Rubio, A.; Barth, J. V.; Narita, A.; Müllen, K.; Berger, R.; Feng, X.; Palma, C.-A.; Auwärter, W. Nat. Commun. 2020, 11, 1490. doi:10.1038/s41467-020-15210-2

19. Nagashima, A.; Tejima, N.; Gamou, Y.; Kawai, T.; Oshima, C. Phys. Rev. Lett. 1995, 75, 3918-3921. doi:10.1103/physrevlett.75.3918

20. Auwärter, W.; Kreutz, T. J.; Greber, T.; Osterwalder, J. Surf. Sci. 1999, 429, 229-236. doi:10.1016/s0039-6028(99)00381-7

21. Corso, M.; Auwärter, W.; Muntwiler, M.; Tamai, A.; Greber, T.; Osterwalder, J. Science 2004, 303, 217-220. doi:10.1126/science.1091979

22. Berner, S.; Corso, M.; Widmer, R.; Groening, O.; Laskowski, R.; Blaha, P.; Schwarz, K.; Goriachko, A.; Over, H.; Gsell, S.; Schreck, M.; Sachdev, H.; Greber, T.; Osterwalder, J. Angew. Chem., Int. Ed. 2007, 46, 5115-5119. doi:10.1002/anie.200700234

23. Herden, T.; Ternes, M.; Kern, K. Nano Lett. 2014, 14, 3623-3627. doi:10.1021/nl501349r

24. Schulz, F.; Drost, R.; Hämäläinen, S. K.; Demonchaux, T.; Seitsonen, A. P.; Liljeroth, P. Phys. Rev. B 2014, 89, 235429. doi:10.1103/physrevb.89.235429

25. Laskowski, R.; Blaha, P.; Schwarz, K. Phys. Rev. B 2008, 78, 045409. doi:10.1103/physrevb.78.045409

26. Schwarz, M.; Riss, A.; Garnica, M.; Ducke, J.; Deimel, P. S.; Duncan, D. A.; Thakur, P. K.; Lee, T.-L.; Seitsonen, A. P.; Barth, J. V.; Allegretti, F.; Auwärter, W. ACS Nano 2017, 11, 9151-9161. doi:10.1021/acsnano.7b04022

27. Joshi, S.; Ecija, D.; Koitz, R.; lannuzzi, M.; Seitsonen, A. P.; Hutter, J.; Sachdev, H.; Vijayaraghavan, S.; Bischoff, F.; Seufert, K.; Barth, J. V.; Auwärter, W. Nano Lett. 2012, 12, 5821-5828. doi:10.1021/nl303170m

28. Siegel, G.; Ciobanu, C. V.; Narayanan, B.; Snure, M.; Badescu, S. C. Nano Lett. 2017, 17, 2404-2413. doi:10.1021/acs.nanolett.6b05409

29. Brülke, C.; Heepenstrick, T.; Humberg, N.; Krieger, I.; Sokolowski, M.; Weiß, S.; Tautz, F. S.; Soubatch, S. J. Phys. Chem. C 2017, 121, 23964-23973. doi:10.1021/acs.jpcc.7b06107

30. Zhang, Q.; Yu, J.; Ebert, P.; Zhang, C.; Pan, C.-R.; Chou, M.-Y.; Shih, C.-K.; Zeng, C.; Yuan, S. ACS Nano 2018, 12, 9355-9362. doi:10.1021/acsnano.8b04444

31. Baykara, M. Z.; Schwarz, U. D. 3D Force Field Spectroscopy. In Noncontact Atomic Force Microscopy; Morita, S.; Giessibl, F. J.; Meyer, E.; Wiesendanger, R., Eds.; NanoScience and Technology, Vol. 3; Springer International Publishing: Cham, Switzerland, 2015; pp 9-28. doi:10.1007/978-3-319-15588-3_2

32. Crommie, M. F.; Lutz, C. P.; Eigler, D. M. Nature 1993, 363, 524-527. doi:10.1038/363524a0

33. De Renzi, V.; Rousseau, R.; Marchetto, D.; Biagi, R.; Scandolo, S.; del Pennino, U. Phys. Rev. Lett. 2005, 95, 046804. doi:10.1103/physrevlett.95.046804

34. Ziroff, J.; Gold, P.; Bendounan, A.; Forster, F.; Reinert, F. Surf. Sci. 2009, 603, 354-358. doi:10.1016/j.susc.2008.11.019

35. Cassabois, G.; Valvin, P.; Gil, B. Nat. Photonics 2016, 10, 262-266. doi:10.1038/nphoton.2015.277

36. Kittel, C.; McEuen, P. Introduction to Solid State Physics; John Wiley \& Sons, 2018.

37. Wandelt, K. Appl. Surf. Sci. 1997, 111, 1-10. doi:10.1016/s0169-4332(96)00692-7

38. Ploigt, H.-C.; Brun, C.; Pivetta, M.; Patthey, F.; Schneider, W.-D. Phys. Rev. B 2007, 76, 195404. doi:10.1103/physrevb.76.195404
39. Ruffieux, P.; Aït-Mansour, K.; Bendounan, A.; Fasel, R.; Patthey, L.; Gröning, P.; Gröning, O. Phys. Rev. Lett. 2009, 102, 086807. doi:10.1103/physrevlett.102.086807

40. Borca, B.; Barja, S.; Garnica, M.; Sánchez-Portal, D.; Silkin, V. M.; Chulkov, E. V.; Hermanns, C. F.; Hinarejos, J. J.; Vázquez de Parga, A. L.; Arnau, A.; Echenique, P. M.; Miranda, R. Phys. Rev. Lett. 2010, 105, 036804. doi:10.1103/physrevlett.105.036804

41. Bokdam, M.; Brocks, G.; Katsnelson, M. I.; Kelly, P. J. Phys. Rev. B 2014, 90, 085415. doi:10.1103/physrevb.90.085415

42. N'Diaye, A. T.; Bleikamp, S.; Feibelman, P. J.; Michely, T. Phys. Rev. Lett. 2006, 97, 215501. doi:10.1103/physrevlett.97.215501

43. Gundlach, K. H. Solid-State Electron. 1966, 9, 949-957. doi:10.1016/0038-1101(66)90071-2

44. Melitz, W.; Shen, J.; Kummel, A. C.; Lee, S. Surf. Sci. Rep. 2011, 66, 1-27. doi:10.1016/j.surfrep.2010.10.001

45. Gómez Díaz, J.; Ding, Y.; Koitz, R.; Seitsonen, A. P.; lannuzzi, M.; Hutter, J. Theor. Chem. Acc. 2013, 132, 1350. doi:10.1007/s00214-013-1350-z

46. Zerweck, U.; Loppacher, C.; Otto, T.; Grafström, S.; Eng, L. M. Phys. Rev. B 2005, 71, 125424. doi:10.1103/physrevb.71.125424

47. Giessibl, F. J. Appl. Phys. Lett. 2000, 78, 123-125. doi:10.1063/1.1335546

48. Ternes, M.; González, C.; Lutz, C. P.; Hapala, P.; Giessibl, F. J.; Jelínek, P.; Heinrich, A. J. Phys. Rev. Lett. 2011, 106, 016802. doi:10.1103/physrevlett.106.016802

49. Giessibl, F. J.; Hembacher, S.; Herz, M.; Schiller, C.; Mannhart, J. Nanotechnology 2004, 15, S79-S86. doi:10.1088/0957-4484/15/2/017

50. Morita, S.; Giessibl, F. J.; Meyer, E.; Wiesendanger, R., Eds. Noncontact Atomic Force Microscopy; NanoScience and Technology, Vol. 3; Springer International Publishing: Cham, Switzerland, 2015. doi:10.1007/978-3-319-15588-3

51. Nečas, D.; Klapetek, P. Cent. Eur. J. Phys. 2012, 10, 181-188. doi:10.2478/s11534-011-0096-2

\section{License and Terms}

This is an Open Access article under the terms of the Creative Commons Attribution License (https://creativecommons.org/licenses/by/4.0). Please note that the reuse, redistribution and reproduction in particular requires that the author(s) and source are credited and that individual graphics may be subject to special legal provisions.

The license is subject to the Beilstein Journal of Nanotechnology terms and conditions: (https://www.beilstein-journals.org/bjnano/terms)

The definitive version of this article is the electronic one which can be found at: https://doi.org/10.3762/bjnano.12.46 